\title{
Long-term sequelae following previous coronavirus epidemics
}

\author{
Author: Oliver O'Sullivan ${ }^{A}$
}

Before the current pandemic, there had been two global epidemics from major coronavirus outbreaks since the turn of the century: severe acute respiratory syndrome coronavirus (SARS-CoV) and Middle East respiratory syndrome coronavirus (MERS-CoV). Both epidemics left survivors with fatigue, persistent shortness of breath, reduced quality of life and a significant burden of mental health problems.

It is likely that some of the chronic problems encountered by survivors of SARS and MERS may be relevant for medical planning of the services required for survivors of coronavirus disease 2019 (COVID-19) caused by the novel coronavirus SARS-CoV-2. Given the similarities between the diseases, the recovery and rehabilitation of the survivors of COVID-19 is likely to be focused around cardiopulmonary sequelae, fatigue and the psychological burden of COVID-19, but in a much larger population.

KEYWORDS: Coronavirus, COVID-19, rehabilitation, rehab

DOI: $10.7861 /$ clinmed.2020-0204

\section{Introduction}

Coronaviruses are large enveloped positive sense RNA viruses causing enteric and respiratory disease. In 2019, there had been two global epidemics from major coronavirus outbreaks since the turn of the century: severe acute respiratory syndrome coronavirus (SARS-CoV) and Middle East respiratory syndrome coronavirus (MERS-COV).

In December 2019, in Wuhan, China, the highly pathogenic SARS-CoV-2 caused a local outbreak of coronavirus disease 2019 (COVID-19), which has now become a well-established global pandemic. COVID-19 has a spectrum of severity, with some patients being asymptomatic and others experiencing short-lived, self-limiting symptoms, but others developing a highly lethal acute pneumonia similar to that seen with SARS-CoV-1 and MERS-CoV. ${ }^{1}$ Genomic analysis has demonstrated that SARS-CoV-2 is in the same beta-coronavirus clade as SARS-CoV-1 and MERS-CoV, and has significant sequence homology with SARS-CoV-1, including

Authors: ${ }^{A}$ specialty registrar in rehabilitation and rheumatology, Defence Medical Rehabilitation Centre Stanford Hall, Stanford-onSour, UK the sequences that mediate their entry through the same surface receptor (angiotensin-converting enzyme 2). ${ }^{2,3}$

While the current focus of management is on the acute phase, attention is shifting to potential post-COVID-19 issues. It is likely that some of the chronic problems encountered by survivors of SARS and MERS may be relevant for medical planning of the services required for survivors of COVID-19. This is especially true for the planning of rehabilitation services given the likely demand in the months and years to come. ${ }^{4,5}$ With that in mind, this paper aims to provide a brief overview of the long-term complications and clinical syndromes encountered by survivors of the previous coronavirus epidemics.

A literature search was performed in early April 2020 using the search terms 'severe acute respiratory syndrome/SARS', 'Middle East respiratory syndrome/MERS', 'recovery', 'complications', 'Iong-term' on PubMed and Google Scholar. Abstracts were reviewed and full texts were sourced when they provided relevant information. Articles were excluded if the full text was not available or it was not written in the English language.

\section{SARS}

SARS was a clinical disease caused by SARS-CoV-1 that emerged in 2003, originating in Foshan, Guangdong region, South China. ${ }^{6}$ As a result of the long incubation period of this disease (10-14 days), it had spread to 29 countries in Asia, Europe and North America by the time awareness of its severity grew, with the largest concentration of North American cases occurring in Toronto, Ontario. By the end of the epidemic, there had been 8,422 cases, with 916 related deaths (case fatality rate [CFR] 11\%). ${ }^{7}$ SARS and its management caused both acute and chronic problems, as noted in multiple follow up studies.

During the acute illness, patients self-reported a median loss of $9 \%$ of their body weight, $67 \%$ reported alopecia (which selfresolved in 6 months) and every mechanically ventilated SARS survivor reported muscle wasting and weakness at the time of discharge. ${ }^{8}$ Myopathy was also reported in $30 \%$ of patients in Hong Kong. ${ }^{9}$ At discharge from hospital, patients complained of immediate complications including new reactive airways diseases, neuropathies, hoarseness following prolonged intubation and heterotopic ossification. 8,10

Tansey et al followed up 117 patients in Toronto who had contracted SARS, with interviews, physical examination, chest radiography, a 6 -minute walk test (6MWT), quality of life (QoL) measures and self-reporting of healthcare utilisation at 3, 6 and 12 months. ${ }^{8}$ They showed that at 1 year, $18 \%$ of individuals had 
a reduced 6MWT due to shortness of breath and fatigue. ${ }^{8} \mathrm{QoL}$ measures (using short form health survey, SF-36) showed a global reduction at 3 months, which had improved but not normalised at 1 year. ${ }^{8}$ Most patients returned to work after a 1-2 month period of reduced hours; however, at 1 year, $17 \%$ of patients hadn't returned and $9 \%$ had not returned to pre-SARS level of work. ${ }^{8}$

A 15-year follow-up study of 80 healthcare workers (HCWs) demonstrated that there were residual radiological lung lesions with corresponding loss of function; however, these did improve within the first year and remained stable thereafter. ${ }^{11}$ The same study also revealed femoral bone necrosis related to the use of high dose steroids in the acute illness. ${ }^{11}$ Another study confirmed residual impairment in ventilation and diffusion capacity for carbon monoxide (DLCO) 2 years after SARS. ${ }^{12}$

Yu et al demonstrated that all age groups had impaired function by following up a paediatric population of 34 individuals with a median age of 14.7 years at 6 and 15 months post SARS infection, with 34 controls (matched for age, sex, race and height). ${ }^{13}$ At 15 months after SARS, absolute and mass-related peak $\mathrm{VO}_{2}$ remained impaired, despite normalisation of lung function. ${ }^{13}$ This reduced aerobic capacity was attributed to impaired perfusion to the lungs at peak exercise and physical deconditioning.

Fatigue, myalgia, depression and poor sleep were seen in a cohort of 22 patients and a post-SARS syndrome, similar to fibromyalgia or post viral chronic fatigue syndrome, was suggested, possibly as a result of the psychological trauma or neurological involvement of SARS. ${ }^{14}$ All bar one of these patients was a HCW.

Mental health problems were common both in the acute illness and long-term. During the acute illness of 101 patients in Wong Tai Sin Hospital in Hong Kong, 27 patients suffered moderate anxiety acutely, with 16 suffering severe anxiety. ${ }^{15}$ At 1-year follow up, there were variable levels of mental health problems in different populations of SARS survivors, with approximately $5 \%$ in one Hong Kong-based study of 75 survivors and $33 \%$ in 117 survivors in Ontario (both measured by SF-36). ${ }^{8,15}$ SARS has been since described as a mental health catastrophe as a result of the psychological impact on survivors, especially in those who were HCWs, including anxiety, depression, psychosis and high rates of post-traumatic stress disorder (PTSD) (up to $44 \%$ in some studies). ${ }^{16}$ There were many reasons suggested for this, including the fatal nature of SARS, family concerns and worries about infecting others. ${ }^{16}$

Improvements were seen following post-SARS rehabilitation. Recovery times in cardiovascular and musculoskeletal performance were improved in 133 SARS survivors in Hong Kong 5 weeks after their acute illness following a 6 -week physical rehabilitation programme. ${ }^{17}$ During follow up of a different population, it was seen that SARS-induced pulmonary lesions recovered to a better extent following rehabilitation.11

While most post-SARS problems settled in time, there were ongoing issues with persistent shortness of breath, fatigue, reduced QoL and a significant burden of mental health problems.

\section{MERS}

MERS first emerged in Jeddah, Saudi Arabia in 2012, and is still circulating, with discrete outbreaks seen in Jeddah, Riyadh and the Republic of Korea. As of 31 January 2020, there had been 2,519 infections with 866 fatalities (CFR 35\%). ${ }^{18}$ Outbreaks often occur in healthcare facilities as a result of delayed recognition leading to secondary infection, and therefore a relatively high number of HCWs have been infected (approximately $20 \%$ of all MERS infections). ${ }^{19}$

As with SARS, presenting symptoms are fever, cough and dyspnoea, although a much higher proportion have gastrointestinal symptoms (up to $50 \%$ present with diarrhoea). ${ }^{20}$ Similar to SARS and COVID-19, the presence of comorbidities and increasing age are associated with poor clinical outcomes..$^{20}$ Most MERS patients present with serious respiratory disease and have abnormalities on chest radiography. ${ }^{21}$ The majority of patients require critical care (up to $89 \%$ in one outbreak) and potentially mechanical ventilation. ${ }^{22}$

Batawi et al followed up 78 MERS survivors with questionnaires at 14 months post-hospitalisation in Saudi Arabia. ${ }^{23}$ Using the SF-36, QoL scores were reduced, with significantly lower scores in those who had had critical care admissions. ${ }^{23}$ Similar to SARS, chronic fatigue symptoms were described in $48 \%$ of survivors at 1 year, reducing to $33 \%$ at 18 months. ${ }^{24} 88 \%$ of MERS survivors were back at work, but the study didn't differentiate on how many were in part- or full-time work. ${ }^{23}$

As seen with SARS survivors, there are high levels of psychiatric disorders, including anxiety, depression and PTSD, notably worse in HCWs. One study showed that at 12 months post-MERS $27 \%$ of survivors had depression and $42 \%$ had PTSD, which improved at 18 months but was still a problem in $17 \%$ and $27 \%$ of survivors respectively. ${ }^{24}$ These effects were increased in HCWs, with a different study in South Korea demonstrating that $57 \%$ of nurses who treated patients with MERS suffered PTSD. ${ }^{25}$

Aside from mental health, there was a notable difference between HCWs and non-HCWs, with less severe disease and better survival rates seen in HCWs. This was likely due to the HCWs being younger and having fewer comorbidities. ${ }^{20}$

The literature is sparse, but the specific rehabilitation demands of patients who have survived MERS are likely to be similar to SARS survivors, and based around fatigue, pulmonary rehabilitation, mental health support and post intensive care management.

\section{Other conditions}

Similar findings to those seen in survivors of SARS and MERS have been seen in survivors of non-coronavirus-related acute respiratory distress syndrome (ARDS). Herridge et al followed ARDS survivors up at 3, 6 and 12 months using questionnaires and basic assessments. ${ }^{26}$ Their findings replicated those seen in survivors of both epidemics, with significant weight loss (up to $18 \%$ of body weight), reduced 6MWT at 1 year, reduced QoL scores and persistent functional disability 1 year after critical care discharge. ${ }^{26}$ They followed this population up for 5 years and demonstrated that exercise limitation, physical sequelae and reduced QoL persisted to that point. ${ }^{27}$

Similar problems can be seen in all those who require critical care. Post intensive care syndrome (PICS) is a common constellation of physical, psychological and cognitive problems experienced by those who have been in critical care, with holistic rehabilitation programmes required for each component. ${ }^{28}$

\section{Conclusion}

Given the similarities noted between SARS, MERS, ARDS and COVID-19, it is reasonable to infer that the complications will be similar with a significant impact on long-term morbidity in a large patient population. Patients recovering from COVID-19 are likely to need support for a variety of complications with individually 
tailored rehabilitation programmes, including cardiopulmonary, psychological and PICS rehabilitation.

Any clinician in primary or secondary care caring for patients who have had COVID-19 will need to monitor for likely long-term complications to help identify any occult problems requiring additional help.

\section{References}

1 Huang C, Wang Y, Li X et al. Clinical features of patients infected with 2019 novel coronavirus in Wuhan, China. Lancet 2020;395:497-506.

2 Yu F, Du L, Ojcius DM et al. Measures for diagnosing and treating infections by a novel coronavirus responsible for a pneumonia outbreak originating in Wuhan, China. Microbes Infect 2020;22:74-9.

3 Lu R, Zhao X, Li J et al. Genomic characterisation and epidemiology of 2019 novel coronavirus: implications for virus origins and receptor binding. Lancet 2020;395:565-74.

4 Coraci D, Fusco A, Frizziero A et al. Global approaches for global challenges: The possible support of rehabilitation in the management of COVID-19. J Med Virol 2020, in press (doi: 10.1002/ jmv.25829).

5 Koh GC-H, Hoenig $\mathrm{H}$. How should the rehabilitation community prepare for 2019-nCoV? Arch Phys Med Rehab 2020;101:1068-71.

6 Hui DSC, Chan MCH, Wu AK, Ng PC. Severe acute respiratory syndrome (SARS): epidemiology and clinical features. Postgrad Med J 2004;80:373-81.

7 Chan-Yeung M, Xu R. SARS: epidemiology. Respirology 2003;8:S9S14.

8 Tansey C, Louie M, Loeb M et al. One-year outcomes and health care utilization in survivors of severe acute respiratory syndrome. Arch Intern Med 2007;167:1312-20.

9 Lee N, Hui D, Wu A et al. A major outbreak of severe acute respiratory syndrome in Hong Kong. N Engl J Med 2003;348:1986-94.

10 Tsai LK, Hsieh ST, Chao CC et al. Neuromuscular disorders in severe acute respiratory syndrome. Arch Neurol. 2004;61:1669-73.

11 Zhang $\mathrm{P}, \mathrm{Li}$ ], Liu $\mathrm{H}$ et al. Long-term bone and lung consequences associated with hospital-acquired severe acute respiratory syndrome: a 15 year follow-up from a prospective cohort study. Bone Research 2020;8:8.

12 Ngai JC, Ko FW, Ng SS et al. The long-term impact of severe acute respiratory syndrome on pulmonary function, exercise capacity and health status. Respirology 2010;15:543-50.

13 Yu CCW, Li AM, So RCH et al. Longer term follow up of aerobic capacity in children affected by severe acute respiratory syndrome (SARS). Thorax 2006;61:240-6.

14 Moldofsky H, Patcai J. Chronic widespread musculoskeletal pain, fatigue, depression and disordered sleep in chronic post-SARS syndrome; a case-controlled study. BMC Neurol 2011;11:37.

15 Chan KS, Zheng JP, Mok YW et al. SARS: Prognosis, outcome and sequelae. Respirology 2003;8:S36-S40.
16 Gardner PJ, Moallef P. Psychological impact on SARS survivors: Critical review of the English language literature. CMAJ 2015:56;123-35.

17 Lau MC, Ng YF, Jones YM et al. A randomised controlled trial of the effectiveness of an exercise training program in patients recovering from severe acute respiratory syndrome. Aus ] Physio 2005;51:213-9.

18 World Health Organization. Middle East respiratory coronavirus (MERS-CoV) - The Kingdom of Saudi Arabia. www.who.int/csr/ don/24-february-2020-mers-saudi-arabia/en/ [Accessed 10 April 2020].

19 Elkholy AA, Grant R, Assiri A et al. MERS-CoV infection among healthcare workers and risk factors for death: Retrospective analysis of all laboratory-confirmed cases reported to WHO from 2012 to 2 June 2018. J Infect Pub Health 2020;13:418-22.

20 Garbati MA, Shamsudeen FF, Fang VJ et al. A comparative study of clinical presentation and risk factors for adverse outcome in patients hospitalised with acute respiratory disease due to MERS coronavirus or other causes. PLoS One 2016;11:e0165978.

21 Assiri A, Al-Tawfiq JA, Al-Rabeeah AA et al. Epidemiological, demographic, and clinical characteristics of 47 cases of Middle East respiratory syndrome coronavirus disease from Saudi Arabia: a descriptive study. Lancet Infect Dis 2013;13:752-61.

22 White J. Middle Eastern Respiratory Syndrome Coronavirus (MERS-CoV). Clin Mic News 2014;36:115-22.

23 Batawi S, Tarazan N, Al-Raddadi R et al. Quality of life reported by survivors after hospitalization for Middle East respiratory syndrome (MERS). Health Qual Life Outcomes 2019;17:101.

24 Lee SH, Shin HS, Park HY et al. Depression as a mediator of chronic fatigue and post-traumatic stress symptoms in Middle East respiratory syndrome survivors. Psychiatry Investig 2019;16:59-64.

25 Jung $\mathrm{H}$, Jung SY, Lee MH et al. Assessing the presence of posttraumatic stress and turnover intention among nurses post-middle east respiratory syndrome outbreak: the importance of supervisor support. Workplace Health Saf 2020;68:337-45.

26 Herridge MS, Cheung AM, Tansey CM et al. One-year outcomes in survivors of the acute respiratory distress syndrome. N Engl J Med 2003:348:683-93.

27 Herridge MS, Tansey CM, Matté A et al. Functional disability 5 years after acute respiratory distress syndrome. N Engl J Med 2011;364:1293

28 Mikkelsen M, Netzer G, Iwashyna T. Post intensive care syndrome (PICS). www.uptodate.com/contents/post-intensive-care-syndromepics [Accessed 5 May 2020].

Address for correspondence: Dr Oliver O'Sullivan, Defence Medical Rehabilitation Centre Stanford Hall, Stanford-on-Soar, Nottinghamshire LE12 5QW, UK.

Email: oliver.o'sullivan@nhs.net

Twitter: @ollieosul 